\title{
Mitral Regurgitation After Orthotopic Lung Transplantation: Natural History and Impact on Outcomes
}

\author{
Sharon L. McCartney, MD*,1, Mary Cooter, MS*, \\ Zainab Samad, MD, MHS ${ }^{\dagger}$, Joseph Sivak, $\mathrm{MD}^{\dagger}$, \\ Anthony Castleberry, $\mathrm{MD}^{\ddagger}$, Stephen Gregory, $\mathrm{MD}^{*}$, \\ John Haney, $\mathrm{MD}^{\ddagger}$, Matthew Hartwig, $\mathrm{MD}^{\ddagger}$, \\ Madhav Swaminathan, MD, MB, BS* \\ *Division of Cardiothoracic Anesthesiology, Department of Anesthesiology, Duke University Medical Center, \\ Durham, NC \\ ${ }^{\dagger}$ Division of Cardiology, Department of Internal Medicine, Duke University Medical Center, Durham, NC \\ ${ }^{\ddagger}$ Division of Cardiothoracic Surgery, Department of Surgery, Duke University Medical Center, Durham, NC
}

\begin{abstract}
Objective: Progression of mitral regurgitation (MR) after orthotopic lung transplantation (OLT) may be an underrecognized phenomenon due to the overlapping symptomatology of pulmonary and valvular disease. Literature evaluating the progression of MR after OLT currently is limited to case reports. Therefore, the hypothesis that MR progresses after OLT was tested and the association of preprocedure MR with postoperative mortality was assessed. Design: A retrospective cohort.

Setting: A tertiary-care hospital.

Participants: Patients who underwent OLT between January 1, 2003 and February 4, 2012.

Interventions: After receiving institutional review board approval, a preprocedure transesophageal echocardiogram was compared with a postoperative transthoracic echocardiogram (TTE) to determine the progression of MR. Univariate and multivariate association between preprocedure MR grade and 1- and 5-year mortality was assessed. A $p$ value of $<0.05$ was considered statistically significant.

Measurements and Main Results: From 715 patients who underwent OLT, 352 had a postoperative TTE and were included in the evaluation of progression of MR. Five patients had progression of MR postoperatively, and the mean change in MR score of -0.04 was found to be nonsignificant $(p=0.25)$. Mortality data were available for 634 of the 715 patients. After covariate adjustment, there was no significant association between MR grade and 1-year mortality $(\mathrm{p}=0.20)$ or 5 -year mortality $(\mathrm{p}=0.46)$.

Conclusions: This study rejected the hypothesis that primary and secondary MR progresses after OLT and found that preprocedure MR was not associated with increased postoperative mortality. Despite the findings that MR does not progress in all patients, there is a subset of patients for whom MR progression is clinically significant.
\end{abstract}

(c) 2017 Elsevier Inc. All rights reserved.

Key Words: lung transplantation; mitral valve insufficiency; mitral valve regurgitation; mitral valve incompetence; mitral insufficiency; mitral incompetence; mitral regurgitation

There was no funding to support this work. There are no commercial or noncommercial conflicts of interest.

${ }^{1}$ Address reprint requests to Sharon McCartney, MD, Box 3094, DUMC Anesthesiology, Erwin Rd, Durham, NC 27710.

E-mail address: Sharon.McCartney@duke.edu (S.L. McCartney).
PATIENTS PRESENTING WITH respiratory compromise after orthotopic lung transplantation (OLT) may be demonstrating progression of pre-existing mitral regurgitation (MR). ${ }^{1-4}$ However, due to the overlapping symptomatology 
of MR and respiratory insufficiency, the diagnosis and therefore treatment of MR may be delayed, thereby increasing morbidity and healthcare use. Determination of the natural course of MR after OLT, with early identification of patients who are most at risk of MR progression, may decrease morbidity and improve pulmonary mechanics.

In the first report of concomitant MR leading to respiratory failure after lung transplantation, Hamad et $\mathrm{al}^{2}$ described a patient with preoperative moderate MR who was unable to be weaned from mechanical ventilation for 3 weeks. Eventually, mitral valve replacement was performed, and the patient was weaned from mechanical ventilation on the third postoperative day. Subsequently, other cases were reported that described new-onset MR that led to respiratory compromise, including a dramatic case of new-onset severe MR that was recognized only after the patient could not be weaned from mechanical ventilation for 42 days after OLT, with successful subsequent extubation on the fifth postoperative day after mitral valve repair. ${ }^{1,3,4}$

One hypothesis attributes worsening of MR to a reduction in size of the right ventricle $(\mathrm{RV})$, producing inverse changes in the left ventricle and mitral annulus. ${ }^{1}$ According to this hypothesis, the primary lung disease causes pulmonary hypertension, which results in RV dilation and dysfunction. The RV dilation then results in a reduction of the size of the mitral valve (MV) annulus. With lung transplantation and resolution of the end-stage lung disease, the severity of pulmonary hypertension decreases over time, leading to improved RV function and decreased RV dilation, which cause a reciprocal conformational change in the MV annulus and unmask the MV pathology and MR. However, the progression of MR after OLT and its prognostic value have not been studied systematically. To determine the natural history of concurrent primary and secondary MR in the OLT population, the hypothesis that the severity of MR worsens after OLT was tested. Additionally, the association of preprocedure MR with mortality was evaluated to identify a cohort who may be at increased risk of mortality after OLT.

\section{Methods}

\section{Study Population}

After receiving approval from the institutional review board, all adult patients who underwent OLT from January 1, 2003 to February 4, 2012 at the authors' institution were identified from an institutional database. Patients receiving combined heart and lung transplantation were excluded due to concomitant heart transplant, making the preprocedure MR incomparable to postprocedure MR. Patients receiving sequential OLTs (ie, a right single-lung transplant followed by a left single-lung transplant) or retransplantation of a bilateral OLT were considered as separate entities each time they presented for transplantation because decompressing effects of the RV leading to reciprocal changes in MV annulus geometry may be more pronounced at the time of the second transplant due to improved oxygenation and decrease in pulmonary vascular resistance.

\section{Demographic Data}

Demographic data were obtained from the institutionally maintained database for patients who had undergone lung transplantation at the authors' university. Demographic variables included age, sex, and race. Additional information regarding the patient's medical history, including the etiology of end-stage lung disease, history of coronary artery disease or percutaneous coronary intervention, and lung allocation score (LAS) also were obtained from this database. The LAS estimates the lung transplant candidate's medical urgency and probability of survival following a lung transplant and is used to determine lung allocation when a potential donor becomes available. ${ }^{5}$

\section{Echocardiographic Data}

Echocardiographic data pertaining to MR were obtained from electronic databases containing detailed intraoperative transesophageal echocardiographic (TEE) information and postoperative transthoracic echocardiographic (TTE) followup examinations. Intraoperative TEE information was derived from reports previously read and interpreted by a cardiothoracic anesthesiologist who was board certified in perioperative TEE. The TTE information was derived from reports previously read and interpreted by a cardiologist who was board certified in transthoracic echocardiography.

Exact parameters used to grade severity of MR (vena contracta, effective regurgitant orifice area, etc.) were not standardized and were determined by the echocardiographer and interpreter; however, the severity of MR was graded using the American Society of Echocardiography guidelines. ${ }^{6,7}$ The grade of MR on the preprocedure intraoperative TEE was compared to the grade of MR on any postoperative TTE closest to 1 year postoperatively. The TTEs performed in the first 7 postoperative days were excluded because this was believed to be a time of rapidly changing hemodynamics that could influence the grade of the MR reported. Patients were excluded from analysis of the primary hypothesis if either the preprocedure TEE or postprocedure TTE was not available. Additional echocardiographic information obtained from these databases included the presence of RV dilation or dysfunction (present/absent, severity not graded) on the preprocedure TEE and MV pathology on the postoperative TTE. The severity of RV dilation or dysfunction was not graded as the American Society of Echocardiography guidelines do not provide distinct quantitative definitions that differentiate severity of $\mathrm{RV}$ dilation or dysfunction (ie, mild, moderate, or severe). ${ }^{8}$

\section{Procedural Data}

All patients who underwent single or bilateral orthotopic lung transplantation were included. Concomitant procedures that were performed at the time of lung transplantation were 
reviewed, including coronary artery bypass grafting or intraaortic balloon pump insertion.

\section{Outcome Data}

Mortality data were obtained from an institutionally maintained database. Mortality was reported at the time of death by the lung transplant coordinator. Patients who underwent lung transplantation at the authors' institution were required to relocate locally and follow closely with the pulmonologist and lung transplant coordinator when significant illnesses or death occurred. To ensure that the mortality data in the database were current at the time of analysis, a secondary chart review of all patients was made to update any deaths since the institutional database was last updated. Cases in which mortality data were not available were limited to patients who had transferred their care to their local pulmonologist after the transplant team had decided they were eligible for this transition. Patients were included in the secondary outcome analysis if preprocedure TEE and mortality outcome data were available.

\section{Statistical Analysis}

The MR grade was analyzed by assigning equally spaced scores to the graded severity as follows: none or trace (0), mild (1), and moderate or severe (2). Moderate and severe MR were grouped together in the analysis because these grades were considered to be of equal clinical significance. The difference between a grade of moderate and a grade of severe MR may be related to alterations in hemodynamics but both are likely to be clinically significant. The primary hypothesis was assessed using a two-sided Wilcoxon signed rank test of the change in MR grade (postoperative TTE MR score - preprocedure TEE MR score). An association between the change in MR grade and the presence of preprocedure RV dysfunction on TEE was assessed using a two-sided chi-square test. A post hoc power analysis of the primary hypothesis was performed with PASS software (NCSS LLC; Kaysville, UT) using 5,000 MonteCarlo simulations of the sample size with the authors' observed variance and a specified clinically meaningful detection margin of 1-2 grade of change to ensure that an appropriate sample size was obtained.

The univariable and LAS-adjusted associations between 1and 5-year mortality and preprocedure MR grade score were assessed using logistic regression models. Additionally, the univariable association between MR grade and the distribution of survival time was assessed via Kaplan-Meier curves and a log-rank trend test. A p value of $<0.05$ was considered significant. All analyses were performed using SAS version 9.4 (SAS Inc., Cary, NC).

\section{Results}

Between January 1, 2003 and February 4, 2012, 715 patients underwent OLT at the authors' institution. Of the 715 patients, 352 patients had complete data pertaining to both preprocedure intraoperative TEE and postoperative follow-up TTE and constituted the final study cohort for the primary analysis. A description of this population is shown in Table 1.

The median time from preprocedure TEE to postprocedure TTE was 58 weeks (interquartile range, 35-108 weeks). A majority of patients had none or trace MR $(n=266 ; 76 \%)$ while a small proportion had moderate or severe $\operatorname{MR}(\mathrm{n}=12$; $3 \%$; Table 2). From the overall cohort, 51 patients $(14 \%)$ had worsening of preprocedure MR, 61 (17\%) patients showed improvement, and MR did not change in the remainder (Table 2). From the cohort of 352 patients, 36 patients had mitral annular calcification, 10 had thickened mitral valve leaflets, 9 had prolapse of one or both mitral valve leaflets, and the remainder of the patients had normal mitral valve morphology. Of the patients with mitral valve prolapse, 8 had trace or mild MR on postoperative TTE and 1 patient had progressed from mild MR on preprocedure TEE to severe MR on postoperative TTE over a period of 170.9 weeks.

Testing of the primary hypothesis revealed a mean (SD) change in overall MR score of $-0.04(0.6)$ and a median (interquartile range) change of $0(0)$, which was found to be nonsignificant $(\mathrm{p}=0.25)$. The annual distribution of the cohort remained relatively stable, except for 2012, in which

Table 1

Demographic and Comorbid Variables of Patient Population

\begin{tabular}{|c|c|}
\hline Variable & $\begin{array}{l}\text { Number of Cases } \\
\text { (Percent) }\end{array}$ \\
\hline \multicolumn{2}{|l|}{ Diagnosis Category } \\
\hline Obstructive disease & $106(30)$ \\
\hline Restrictive disease & $179(51)$ \\
\hline Cystic fibrosis/bronchiectasis & $49(14)$ \\
\hline Primary pulmonary hypertension & $4(1)$ \\
\hline Other & $14(4)$ \\
\hline \multicolumn{2}{|l|}{ Sex } \\
\hline Female & $140(40)$ \\
\hline Male & $212(60)$ \\
\hline \multicolumn{2}{|l|}{ Race } \\
\hline Caucasian & $315(90)$ \\
\hline Asian & $2(1)$ \\
\hline African American & $29(8)$ \\
\hline Other & $6(2)$ \\
\hline \multicolumn{2}{|l|}{ Diabetes } \\
\hline Yes & $60(17)$ \\
\hline History of congestive heart failure & $27(8)$ \\
\hline \multicolumn{2}{|l|}{$\begin{array}{l}\text { History of coronary artery disease (revascularized or } \\
\text { insignificant) }\end{array}$} \\
\hline Yes & $113(32)$ \\
\hline History of PCI & $18(5)$ \\
\hline History of CABG & $3(1)$ \\
\hline $\begin{array}{l}\text { Underwent concurrent } \mathrm{CABG} \text { at time of lung } \\
\text { transplantation }\end{array}$ & $22(6)$ \\
\hline Pulmonary hypertension & $185(53)$ \\
\hline Right ventricular dysfunction on preprocedure TEE & $203(58)$ \\
\hline Age at transplant $(y)($ mean $\pm S D)$ & $55.4(14.3)$ \\
\hline LAS $($ mean \pm SD) & $47.5(16.5)$ \\
\hline $\begin{array}{l}\text { Pulmonary artery mean pressure }(\mathrm{mmHg}) \\
(\text { mean } \pm \mathrm{SD})\end{array}$ & $26.5(9.3)$ \\
\hline
\end{tabular}

Abbreviations: CABG, coronary artery bypass graft; LAS, lung allocation score; PCI, percutaneous coronary intervention. 
Table 2

Comparison of Preprocedure TEE MR Grade With Postprocedure TTE MR Grade

\begin{tabular}{lccccc}
\hline & \multicolumn{5}{c}{ TTE MR Grade } \\
\cline { 3 - 6 } & & $\begin{array}{c}\text { 0: None/ } \\
\text { Trace }\end{array}$ & 1: Mild & $\begin{array}{c}\text { 2: Mod/ } \\
\text { Severe }\end{array}$ & Total \\
\hline TEE MR & 0: None/ & 219 & 46 & 1 & 266 \\
Grade & Trace & & & & \\
& 1: Mild & 53 & 17 & 4 & 74 \\
& 2: Mod/ & 4 & 4 & 4 & 12 \\
& Severe & & & & \\
& Total & 276 & 67 & 9 & 352 \\
\hline
\end{tabular}

Abbreviations: MR, mitral regurgitation; TEE, transesophageal echocardiogram; TTE, transthoracic echocardiogram.

only the data from January 1 to February 4 were included (Tables 3 and 4). Among the 351 patients who also had preprocedure TEE information regarding RV dysfunction or dilation, 203 patients had preprocedure RV hypokinesis or dilation whereas 148 patients had normal RV function and no dilation. Of the patients with RV hypokinesis or dilation, 26 (12.8\%) had a worsening of MR grade, $143(70.4 \%)$ had an MR grade that stayed the same, and 34 (16.8\%) improved. No significant association between the change in MR grade and preprocedure RV dysfunction or dilation $(\mathrm{p}=0.57)$ was found.

Mortality data and preprocedural MR grade on TEE were available for 634 patients. Eighty-eight deaths occurred within 1 year and 237 deaths occurred within 5 years of transplantation. There was evidence of a univariate association between severity of MR and 1-year mortality (odds ratio $[\mathrm{OR}]=1.54$; confidence interval $[\mathrm{CI}]$ 1.02-2.34; $\mathrm{p}=0.04$ ). However, after adjusting for LAS, the association was no longer significant $(\mathrm{OR}=1.35$; CI $0.86-2.12 ; \mathrm{p}=0.20)$. The log-rank test for 1 year survival also showed a significant univariate trend in time to event by MR grade ( $\mathrm{p}=0.03$; Fig 1$)$. There was no significant univariate association between MR grade and 5-year mortality $(\mathrm{OR}=0.96$; CI $0.68-1.34 ; \mathrm{p}=0.79)$, and there was no association after adjusting for LAS (OR $=0.87$; CI 0.60 $1.25 ; \mathrm{p}=0.46)$. The log-rank test for the trend in the 5-year survival found no significant association between the distribution of 5-year survival and MR grade $(p=0.78$; Fig 2).

Table 3

Distribution of the Study Cohort by Year (Patient Distribution by Year)

\begin{tabular}{lcrcc}
\hline Year of Surgery & Count & Percent & Cumulative Count & Cumulative Percent \\
\hline 2003 & 21 & 5.97 & 21 & 5.97 \\
2004 & 34 & 9.66 & 55 & 15.63 \\
2005 & 32 & 9.09 & 87 & 24.72 \\
2006 & 35 & 9.94 & 122 & 34.66 \\
2007 & 29 & 8.24 & 151 & 42.9 \\
2008 & 38 & 10.80 & 189 & 53.69 \\
2009 & 43 & 12.22 & 232 & 65.91 \\
2010 & 48 & 13.64 & 280 & 79.55 \\
2011 & 69 & 19.60 & 349 & 99.15 \\
2012 & 3 & 0.85 & 352 & 100.00 \\
\hline
\end{tabular}

Table 4

Distribution of the Study Cohort by Year (Patient Distribution of MR Severity Change by Year)

\begin{tabular}{lcccr}
\hline \multirow{2}{*}{ Surgery Year } & \multicolumn{4}{c}{ MR Grade Change } \\
\cline { 2 - 5 } & Improves & Stays the Same & Worsens & Total \\
\hline 2003 & $1(4.76)$ & $17(80.95)$ & $3(14.29)$ & 21 \\
2004 & $3(8.82)$ & $27(79.41)$ & $4(11.76)$ & 34 \\
2005 & $5(15.63)$ & $20(62.5)$ & $7(21.88)$ & 32 \\
2006 & $11(31.43)$ & $19(54.29)$ & $5(14.29)$ & 35 \\
2007 & $5(17.24)$ & $19(65.52)$ & $5(17.24)$ & 29 \\
2008 & $9(23.68)$ & $25(65.79)$ & $4(10.53)$ & 38 \\
2009 & $5(11.63)$ & $30(69.77)$ & $8(18.6)$ & 43 \\
2010 & $10(20.83)$ & $29(60.42)$ & $9(18.75)$ & 48 \\
2011 & $10(14.49)$ & $53(76.81)$ & $6(8.7)$ & 69 \\
2012 & $2(66.67)$ & $1(33.33)$ & $0(0)$ & 3 \\
Total & 61 & 240 & 51 & 352 \\
\hline
\end{tabular}

Abbreviations: MR, mitral regurgitation.

\section{Discussion}

In this study, it was found that lung transplantation did not predict worsening of MR. Additionally, the degree of MR at the time of OLT was not associated with increased long-term mortality after controlling for other patient risk factors. From a mechanistic perspective, the presence of preprocedure RV dysfunction was not associated independently with change in MR severity after OLT, questioning the proposed mechanism of MR progression. This was the first study to report the natural history of MR and its association with long-term mortality in the lung transplant population.

Progression of MR after OLT has been found to be significant in a few published case reports. ${ }^{1-4}$ In these reports, prolonged mechanical ventilation or oxygen dependency led to further investigation and unmasking of significant valvular pathology. Recognition of the progression of MR occurred within the first 3 months postoperatively, indicating a unique postoperative phenomenon. In all but one report, surgical correction resulted in prompt discontinuation of mechanical ventilation and oxygen requirements.

Despite the existing case reports, progression of MR appears to be an uncommon issue in this population, with no pattern of change after surgery or mechanistic factor identified. The authors did not find that MR worsened in severity overall, and a predictive value of preprocedure intraoperative MR grade on severity of postoperative follow-up MR was not found. A large majority of the cohort did not have significant MR (none, trace, or mild MR $=340$ / 352 ; 97\%), which was determined by preprocedure intraoperative TEE prior to the transplantation procedure. Progression of MR in this population would either follow the trend in the general population or be affected specifically by the OLT surgery. The findings suggested that a lung transplant procedure did not significantly increase or unmask the severity of primary or secondary MR. In the small proportion of patients who had worsening of MR grade, it was unclear what factors contributed to the progression of MR. 


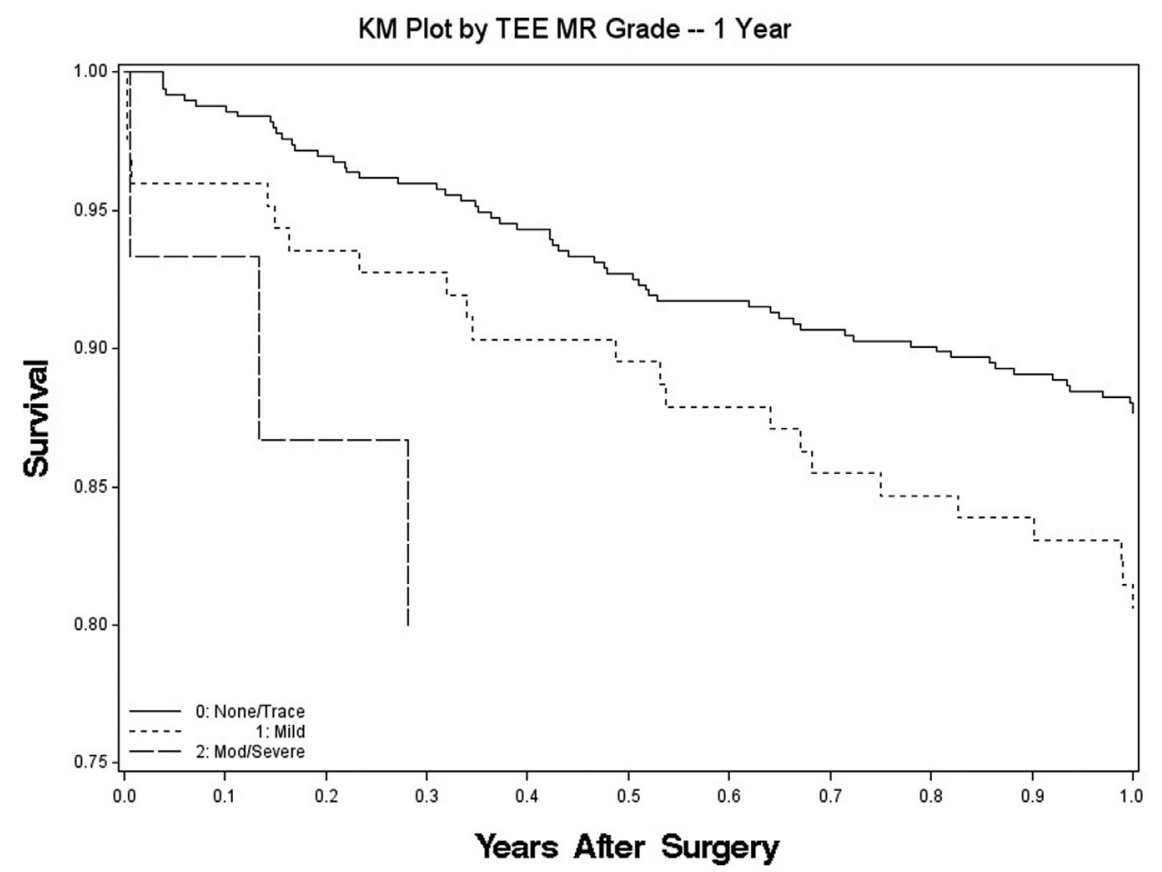

Fig 1. Kaplan-Meier curve of the distribution of 1-year mortality by preprocedure MR grade assessed by TEE. Abbreviations: MR, mitral regurgitation; TEE, transesophageal echocardiography.

In a previous case report, it was found that worsening of MR could be attributed to a reduction in RV internal dimensions after lung transplantation. ${ }^{4}$ The change in RV geometry could have influenced reciprocal changes in the left ventricle, thereby unmasking pre-existing MR and demonstrating a worsening progression postoperatively. However, the results from the current study questioned this hypothesis. No significant association between preprocedure RV dysfunction and postoperative worsening of MR in a systematic analysis was found.

The other significant finding was that there was no significant association between preprocedure MR and longterm mortality following OLT. The trend that was observed in the univariate analysis of 1-year mortality suggested the utility of MR grade severity as a prognostic factor for poor survival, but with only 13 patients and 3 mortalities in the

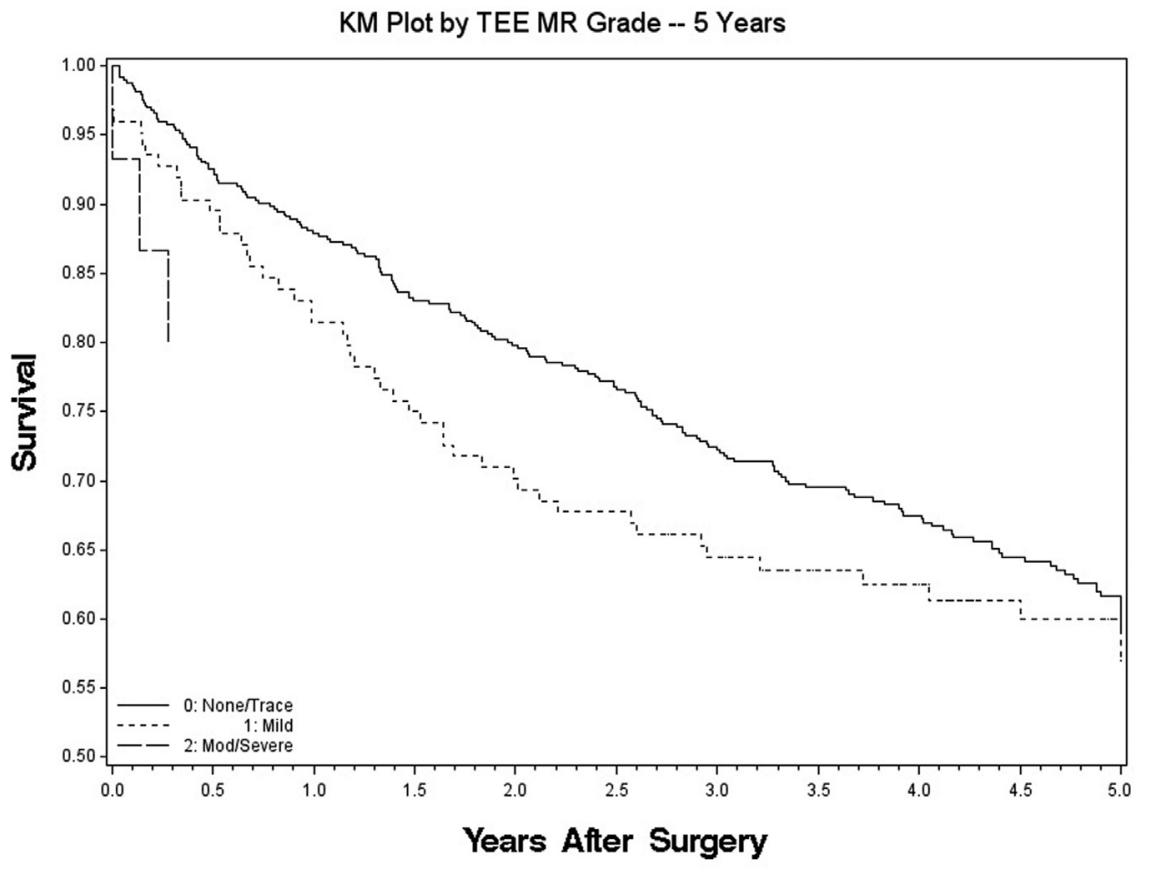

Fig 2. Kaplan-Meier curve of the distribution of 5-year mortality by preprocedure MR grade assessed by TEE. Abbreviations: MR, mitral regurgitation; TEE, transesophageal echocardiography. 
moderate-to-severe MR grade category. A larger study is necessary to establish MR grade as an independent risk factor in this patient population. It is likely that factors other than MR have stronger influences on post-OLT outcomes. Additionally, the patients undergoing OLT who were included in the analysis had a mean pulmonary artery pressure of $26.5 \mathrm{mmHg}$. This degree of pulmonary hypertension was mild for such a population and may have contributed to the overall negative result. It is possible that if a subgroup analysis were performed on patients with moderate-to-severe pulmonary hypertension and/or moderate-to-severe RV dilation and dysfunction, lung transplantation may reduce the internal diameter of the RV in these patients and progression of MR may be evident. While a subgroup analysis in this dataset based on degree of pulmonary hypertension was possible, severity of RV dilation and dysfunction were not available, leading to an incomplete subgroup analysis.

Although its large study population and quality echocardiographic data strengthened this study, there were some limitations. First, preprocedure intraoperative TEE data were compared to postoperative follow-up TTE data. The hemodynamic conditions of these assessments are different, which could influence the degree of MR. During the preprocedure intraoperative TEE assessment, hemodynamics may be influenced by general anesthesia and positive-pressure ventilation; whereas during the TTE assessment, these conditions may not exist. Previous studies have shown that MR usually is downgraded under anesthesia due to reduced afterload. ${ }^{9,10}$ It is therefore even more likely that postoperative TTE would demonstrate worsening of MR simply because of a change in hemodynamic conditions. TEE provides higher resolution and the proximity to the mitral valve may make vena contracta measurements more accurate compared to TTE. ${ }^{6}$ Additionally, regurgitant jet size is affected by transducer frequency and pulse repetition frequency, which may make the regurgitant jet appear larger on TEE compared to TTE. ${ }^{6}$

The study also was limited by cohort data availability because only 352 patients had postoperative TTE assessments; therefore, the sample size decreased significantly from the original 715 who underwent OLT. Given this sample size, however, a post hoc power analysis simulation of a two-sided Wilcoxon signed rank test with type- 1 error rate of 0.05 and the authors' observed variance (0.36) indicated that a similar analysis would be powered adequately (at $>90 \%$ ) to detect a clinically meaningful 1-to-2 degree change in median MR grade (ie, from trace to mild or from trace to moderate). Despite the reduced sample size, in the absence of a single large dataset or multi-institutional dataset including echocardiographic information, this study suggested that the effect, if any, of OLT on preprocedure MR likely was small. Of the 352 patients included in the analysis, only 9 patients had primary MR. Due to the small cohort with primary MR, both primary and secondary MR were combined in the final analysis. This was a limitation of the small sample size. However, if the hypothesis previously made by Bermudez et $\mathrm{al}^{1}$ were taken into consideration, unmasking of $\mathrm{MR}$ due to mitral annular dilation after OLT should affect the severity of MR for both primary and secondary MR.

There also may have been a selection bias in the patients studied. For inclusion in the study, a postprocedure TTE was required. It therefore was likely that a change in symptoms prompted a TTE examination. Additionally, the postprocedure TTE must have been performed within the institution (inpatient or outpatient), and survival to TTE was required for the postprocedure TTE to be captured in the dataset. This study cohort therefore likely excluded patients in extreme circumstances-both asymptomatic patients and those patients who may have been in cardiogenic extremis and did not survive to TTE. However, the potential for asymptomatic patients to have clinically significant progression of MR remains unlikely. The TTE evaluation of patients after cardiothoracic surgery can be limited by poor sound transmission and limited windows, which may affect the grade of postoperative MR observed by TTE. Lastly, although lung transplantation has been performed at the authors' institution by a limited number of surgeons, who also are involved in postoperative management, and longterm care is managed by dedicated pulmonologists subspecializing in lung transplantation, there may be variances in care paradigms that can confound outcomes.

In summary, no evidence that preprocedure MR worsens after OLT and did not significantly influence postoperative mortality in OLT patients was found. Additionally, pretransplant RV dysfunction did not appear to confer added risk of worsening of MR. Although limited by sample size for this hypothesis, the authors are confident that the effect of OLT on MR detected intraoperatively was likely small and that factors other than MR are more likely to contribute to adverse outcome. Future studies should prospectively evaluate larger populations of patients receiving lung transplantation with detailed echocardiographic data. Detailed analysis of RV internal dimensions and mitral annular dimensions with consistent measurements made by one echocardiographer would help to improve the validity of the echocardiographic data. Additionally, as TEE is better suited to grade MR, comparing intraoperative TEE to postoperative TEE at a specific postoperative time point would decrease variability in imaging. It is possible that there are specific subgroups of lung transplant recipients who are at higher risk of progression of MR. These subsets should be identified and subjected to either a modified surgical plan or comprehensive echocardiographic surveillance in the postoperative period.

\section{References}

1 Bermudez CA, Rocha RV, Katz WE, et al. Novel mechanism of mitral regurgitation after lung transplantation in a patient with scleroderma and pulmonary hypertension. J Thorac Cardiovasc Surg 2011;141:e1-3.

2 Hamad AM, Rizzardi G, Bottio T, et al. A word of caution for patients undergoing lung transplantation with associated mitral regurgitation. J Heart Lung Transplant 2008;27:935-6.

3 Udoji TN, Force SD, Pelaez A. Severe mitral regurgitation unmasked after bilateral lung transplantation. Pulm Circ 2013;3:696-9. 
4 McCartney SL, Colin BJ, Duane Davis R, et al. Pulmonary dysfunction after lung transplantation: The dilemma of coexisting mitral regurgitation. J Cardiothorac Vasc Anesth 2014;28:1696-9.

5 Egan TM, Murray S, Bustami RT, et al. Development of the new lung allocation system in the United States. Am J Transplant 2006;6:1212-27.

6 Zoghbi WA, Enriquez-Sarano M, Foster E, et al. Recommendations for evaluation of the severity of native valvular regurgitation with two-dimensional and Doppler echocardiography. J Am Soc Echocardiogr 2003;16:777-802.

7 Lancellotti P, Tribouilloy C, Hagendorff A, et al. Recommendations for the echocardiographic assessment of native valvular regurgitation: An executive summary from the European Association of Cardiovascular Imaging. Eur Heart J Cardiovasc Imaging 2013;14:611-44.
8 Rudski LG, Lai WW, Afilalo J, et al. Guidelines for the echocardiographic assessment of the right heart in adults: A report from the American Society of Echocardiography endorsed by the European Association of Echocardiography, a registered branch of the European Society of Cardiology, and the Canadian Society of Echocardiography. J Am Soc Echocardiogr 2010;23:685-713.

9 Grewal KS, Malkowski MJ, Piracha AR, et al. Effect of general anesthesia on the severity of mitral regurgitation by transesophageal echocardiography. Am J Cardiol 2000;85:199-203.

10 Bach DS, Deeb GM, Bolling SF. Accuracy of intraoperative transesophageal echocardiography for estimating the severity of functional mitral regurgitation. Am J Cardiol 1995;76:508-12. 